\title{
Red-green-blue polymer light-emitting diode pixels printed by optimized laser-induced forward transfer
}

\author{
James Shaw Stewart, ${ }^{1,2, a)}$ Thomas Lippert, ${ }^{2, b)}$ Matthias Nagel, ${ }^{1}$ Frank Nüesch, ${ }^{1,3, c)}$ \\ and Alexander Wokaun ${ }^{2}$ \\ ${ }^{1}$ Laboratory for Functional Polymers, EMPA Swiss Federal Laboratories for Materials Science \\ and Technology, Überlandstrasse 129, CH-8600 Dübendorf, Switzerland \\ ${ }^{2}$ Materials Group, General Energies Department, Paul Scherrer Institut, CH-5232 Villigen-PSI, Switzerland \\ ${ }^{3}$ Section de science et génie des matériaux, EPFL, CH-1015, Lausanne, Switzerland
}

(Received 30 March 2012; accepted 26 April 2012; published online 15 May 2012)

\begin{abstract}
An optimized laser-induced forward transfer (LIFT) technique has been used to fabricate tri-color organic light-emitting diode (OLED) pixels. At reduced pressures, and with a defined donor-receiver gap, patterned depositions of polyfluorene-based OLED pixels have been achieved. OLED pixel functionality has been demonstrated and compared with devices made using conventional deposition techniques. In addition, improved functionality has been obtained by coating the cathode with an electron-injecting layer, a process not possible using conventional OLED fabrication techniques. The OLED pixels fabricated by LIFT reach efficiencies on the range of conventionally fabricated devices and even surpass them in the case of blue pixels. () 2012 American Institute of Physics. [http://dx.doi.org/10.1063/1.4717463]
\end{abstract}

Laser-induced forward transfer (LIFT), also known as laser direct-write, ${ }^{1}$ is a class of printing techniques that have already been used to fabricate basic small-molecule organic light-emitting diodes (OLEDs) $)^{2,3}$ and polymeric OLEDs (PLEDs). ${ }^{4,5}$ OLEDs are a form of solid-state lighting, under intense research for commercial applications, ${ }^{6,7}$ with OLED electronic displays of particular interest. ${ }^{8}$ One challenge which has proved more complex for thin-film electroluminescent (EL) OLEDs than existing liquid crystal display (LCD) technologies is the actual deposition of individual pixels with traditional lithographic techniques requiring specific chemical modification. ${ }^{9}$ Thermal evaporation through a fine metal mask (FMM) is the most reliable mechanism of OLED material deposition, but is only applicable to evaporable materials, generally not polymers. Standard solutionbased printing techniques have been well investigated, ${ }^{10,11}$ but are "wet" processes that require solvent orthogonality. "Dry" OLED printing techniques using lasers have been developed to overcome these problems, ${ }^{8,12-14}$ including LIFT. $^{4,5}$

In our modification of the LIFT process, a thin photodecomposable triazene polymer layer (TP) acts as sacrificial "dynamic release layer" (DRL). ${ }^{15}$ It decomposes under nspulsed UV laser irradiation and propels the overlying layers from the donor to the receiver substrate. Here, we transfer a multilayer stack, comprising the aluminium cathode and the light-emitting polymer (LEP) layer, to the receiver substrate, which consists of indium-doped tin oxide (ITO) coated with hole-transporting layers (HTLs). For the LEP, a single conjugated polymer system of PFO is used. ${ }^{16}$ When doped with triplet emitters, the EL emission color can be changed from

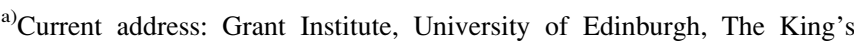
Buildings, Edinburgh EH9 3JW, United Kingdom. Electronic mail: james.shaw-stewart@ed.ac.uk.

${ }^{b)}$ Electronic mail: thomas.lippert@psi.ch.

${ }^{c)}$ Electronic mail: frank.nueesch@empa.ch.
}

blue to green or red. ${ }^{17,18}$ The LIFT deposited devices are referred to as a LIFTed pixels to differentiate them from conventionally fabricated devices.

This research builds on previous depositions of PLED pixels using our variant of LIFT. ${ }^{4,5}$ In particular, the introduction of a gap between the substrates was viewed as a means to improve the robustness and applicability of the technique. LIFT with the substrates "in contact" limits the application of multiple stages of LIFT to the same receiver substrate. The main problem identified with transfer "in contact" was the difficulty of separating the substrates reliably and reproducibly. In addition, the negative consequences of a variable gap width depending on the flatness of the substrates and the cleanness of the surfaces were inferred from poor experimental results. ${ }^{19}$ For these reasons, work into transfer across a well-defined gap was developed and the advantage of reduced pressure for LIFT across a gap was identified and implemented. ${ }^{19}$ In addition, a gap between substrates is more favourable for implementation of LIFT as an industrial manufacturing process, allowing for a faster turnover of substrates. Another improvement which this work demonstrates is the addition of polymeric layers onto the receiver substrate to improve adhesion. ${ }^{5}$ The combination of the reduced pressure and improved interfacial adhesion means that lower fluences are required to transfer pixels, good both in terms of energy usage and for reducing the photon, thermal and mechanical energy load on the transferred pixel.

The architectures of the LIFT donor and receiver substrates are summarized in Figs. 1(a) and 1(b). Suprasil ${ }^{\mathbb{B}}$ fused silica was used for the donor substrate, and ordinary glass, pre-patterned with ITO (140 nm thick, $20 \Omega \square^{-1}$ ) was used for the receiver. Both the substrates are of the same dimensions $(\approx 25 \times 25 \times 1 \mathrm{~mm})$ and were cleaned in the same way using ultrasonic baths of acetone, ethanol, basic cleaning solution, and water, before UV-ozone treatment followed by final baths of water. The receiver substrates were 
(a) Donor Substrate

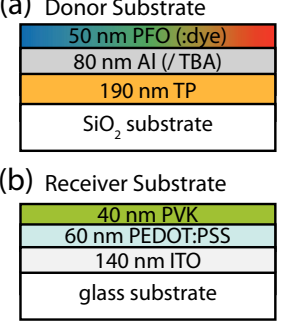

(d)

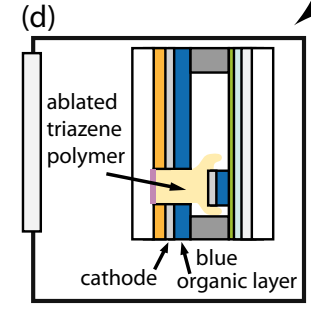

(f)

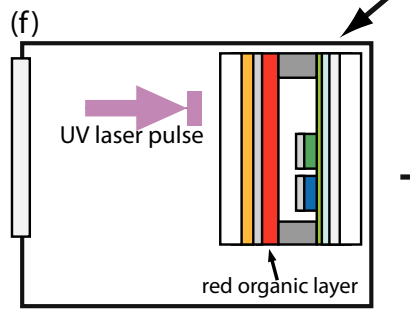

FIG. 1. This scheme summarizes the LIFT process for tri-color OLED pixels. The 1-D substrate architecture is shown for the LIFT donor substrate (a) and the receiver substrate (b). The transfer of the first OLED color, blue, is shown in detail with the laser beam approaching (c) and the TP ablation and pixel deposition (d). LIFT of the green (e) and red (f) OLEDs is shown in sequence, and EL operation of the final three colors, side-by-side is also shown $(\mathrm{g})$.

then spin-coated with $60 \mathrm{~nm}$ of poly[3,4-ethylene dioxythiophene] blended with poly[styrene sulfonate] (PEDOT:PSS, Clevios ${ }^{\mathrm{TM}} \mathrm{P} \mathrm{Al} 4083$ ) and $40 \mathrm{~nm}$ of poly[N-vinylcarbazole] (PVK, $M_{w}=1100000$, Sigma Aldrich). The donor substrates were spin-coated with $190 \mathrm{~nm} \mathrm{TP}$, synthesized according to the procedure for polymer TP-6a previously published in Ref. 20. The aluminium was then evaporated on top of the TP at a vacuum lower than $5 \times 10^{-6} \mathrm{mbar}$, with a deposition rate of $2 \AA \mathrm{s}^{-1}$. Where applied, tetrabutylammonium hydroxide (TBA) was spin-coated at $1500 \mathrm{rpm}$, from a $10^{-4} \mathrm{M}$ methanol solution, to create an ultra-thin layer on the $\mathrm{Al}$ cathode. The LEP was either plain poly[9,9-dioctylfluorenyl-2,7-diyl] capped with silsesquioxane (PFO) for blue emission, or PFO doped with 5 wt. \% iridium(III) tris-(2-(4totyl)pyridinato- $\mathrm{N}, \mathrm{C}^{2}$ ) for green emission, or PFO doped with 5 wt. \% iridium(III) bis-(2-(2'-benzo-thienyl)pyridinato $\mathrm{N}, \mathrm{C}^{3^{\prime}}$ )(acetyl-acetonate) for red emission. All of the LEP materials were bought from ADS. For the LIFT donor substrates, a $50 \mathrm{~nm}$ LEP films was spin-coated onto the cathode, from a toluene:p-xylene (1:1) solution. For the LIFT experiments, single pulses from a XeCl excimer laser $(\lambda=308 \mathrm{~nm}$, $\tau=30 \mathrm{~nm}$ ) have been used. The beam was shaped through a rectangular aperture and the image of the rectangle was focused using an achromatic lens to create a uniform beam of $1.7 \times 0.5 \mathrm{~mm}$ on the donor substrate. The transfers were carried out inside a chamber at a pressure of $1 \mathrm{mbar}$.

The conventionally fabricated devices were fabricated in the same way as the LIFT receiver substrates, but the LEP layer was then spin-coated on top of the PVK. About $40 \mathrm{~nm}$ aluminium or calcium was evaporated on top through a mask to make devices with a circular area of either 3.5 or $7 \mathrm{~mm}^{2}$. From the deposition of the LEP, all of the samples were kept in an inert nitrogen environment and only exposed to air briefly when transferred into the reduced-pressure chamber for the LIFT experiments. All spin-coated film thicknesses were measured using a profilometer (Ambios XP-1), and evaporated film thicknesses were measured using a calibrated quartz-crystal microbalance. The devices were kept in an inert nitrogen glove-box environment and tested in a home-built sample holder with contact pins for applying the bias. The cathodes were connected to the contact pin by evaporating silver and helped with silver paste. It would be possible to make these contact connections by LIFT, and there is research into using direct-write laser deposition for interconnect applications. ${ }^{21}$ A Keithley 2400 sourcemeter was used for the electrical characterization, and this was linked to a Minolta LS-110 light-meter using a home-built LabVIEW program. EL spectra were obtained using a Jobin Yvon Horiba FL-311 Fluorolog, and light microscopy images were taken using a Zeiss Axioplan.

Three different donor substrates are used with the blue, green, and red LEP layers on them. Fig. 1 summarizes the LIFT process with all three depositions required to create three coloured pixels side-by-side. The first step (1c) is shown in detail, where the UV laser pulse is approaching the donor substrate consisting of a UV-transparent substrate coated with, first, a layer of the photolabile triazene polymer DRL, second, the Al cathode layer, and finally, the LEP layer. A second type of pixel was created by spin-coating an ultra-thin layer of the alkaline TBA directly onto the aluminium to help electron injection. ${ }^{22}$ The surface modification of the metal cathode in this way is only possible due to the inverse structure of the donor substrate fabrication (compared with conventionally fabricated devices). The receiver substrate is made up of a glass slide coated with a patterned ITO layer and HTLs which aid the hole injection into the LEP. Both substrates were prepared in and stored in an inert environment and then placed together, with a spacer, in a vacuum chamber for the laser experiments. Fig. 1(d) shows the laser beam being absorbed by the photo-labile triazene DRL, which decomposes to gaseous products that generate a pressure force on the overlying layers and transfer them to the receiver substrate. The $\mathrm{Al}$ (/TBA)/LEP multilayer is propelled across a gap of $15 \mu \mathrm{m}$, defined by a spacer, and deposited onto the receiver substrate at a pressure of $1 \mathrm{mbar}$. Figs. 1(e) and 1(f) show the use of different coloured donor substrates, with Fig. 1(g) illustrating the final result, electroluminescent tri-coloured pixels, side-by-side. The pixels have a width of $500 \mu \mathrm{m}$, but smaller sizes as low as $20 \mu \mathrm{m}$ should be possible, providing the high resolution required for 300 ppi high-definition (HD) displays for handheld electronic devices.

Figs. 2(a) and 2(b) show light microscopy images of three colored pixels, transferred side-by-side and illuminated by applying biases across the devices. An important feature of these devices is the color contrast induced by the different doping of the LEP layer, which is also backed-up by the EL spectra in Figs. 2(c) and 2(d). Figs. 2(c) and 2(d) show the EL spectra of the LIFTed pixels with and without TBA, 


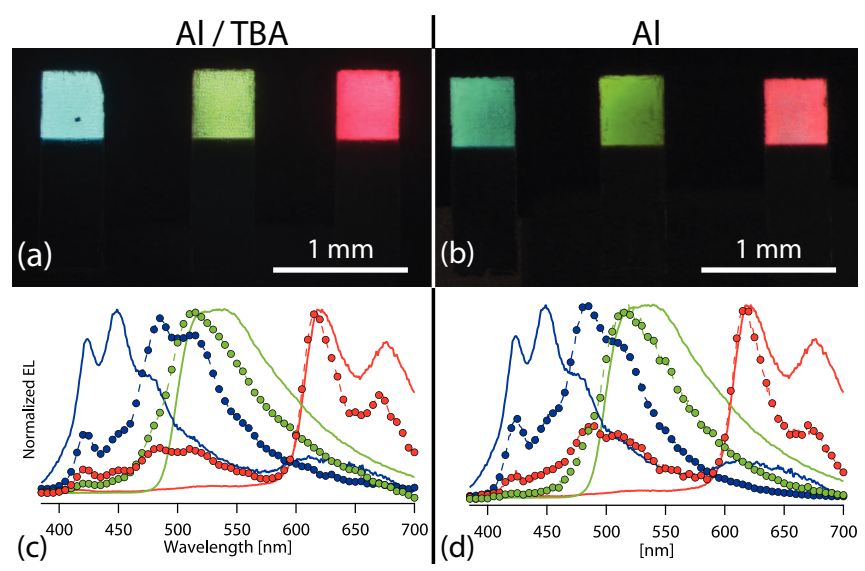

FIG. 2. Tri-color pixels imaged using a light microscope are shown for an $\mathrm{Al} / \mathrm{TBA}$ cathode at $20 \mathrm{~V}$ (a) and for an $\mathrm{Al}$ cathode at $24 \mathrm{~V}$ (b). Electroluminescence (EL) spectra of tri-color LIFT pixels with an Al/TBA cathode (c), and with an Al cathode (d) are shown with dashed lines and circular markers. On both graphs (c) and (d), the Al conventionally fabricated devices' EL spectra are shown with solid lines. The color of the EL spectrum corresponds to the device color.

respectively, with the EL spectra of the conventionally fabricated devices in the background. The EL spectra of the LIFTed red and green pixels are not hugely different to the conventionally fabricated devices, but the blue devices show a significant shift towards green emission. Even if the two blue LIFTed pixels look slightly different, the EL spectra reveal that the spectral emission is actually the same. Any differences between the appearance of the pixel colors in Figs. 2(a) and 2(b) can be explained by differences in relative brightness. High wavelength emission of PFO has been investigated in detail by others, and the accepted explanation is triplet emission from oxidized keto-defects, but the mechanisms for the ketone formation are not well understood. ${ }^{23}$ However, thermal annealing of the conventional devices caused no change in the blue EL spectrum, suggesting that the process may be linked to the fast dynamics of the LIFT process.

In addition to the main advantages of patterned directwrite deposition of functional OLEDs across a gap, a peculiar advantage of the LIFT process stems from the inverse order of the donor substrate layer deposition in contrast with conventionally fabricated devices. The LEP is deposited on top of the cathode, allowing for direct cathode surface modification. From Table I, the addition of TBA onto the aluminium does not change the operating voltages much, but it does significantly increase both the luminous efficiency (LE) and the external quantum efficiency (EQE) by about $50 \%$ for all
TABLE I. Device performances for various device architectures, at a CD of $\sim 40-50 \mathrm{~mJ} \mathrm{~cm}^{-2}$. Blue, green, and red refer to the LEP layer, whether just plain PFO (blue), or doped PFO (red and green). The LIFT Al are the LIFTed pixels with plain Al electrodes, LIFT Al/TBA are the LIFTed pixels with $\mathrm{Al} /$ tetrabutylammonium electrodes, the Convent $\mathrm{Al}$ are the conventionally fabricated devices with $\mathrm{Al}$ electrodes, and Convent $\mathrm{Ca}$ are the conventionally fabricated devices with Ca electrodes.

\begin{tabular}{|c|c|c|c|c|c|c|}
\hline & & $\begin{array}{c}\text { Bias } \\
(\mathrm{V})\end{array}$ & $\begin{array}{c}\mathrm{CD} \\
\left(\mathrm{mJ} \mathrm{cm}^{-2}\right)\end{array}$ & $\begin{array}{c}\text { Lum } \\
\left(\mathrm{cd} \mathrm{m}^{-2}\right)\end{array}$ & $\begin{array}{c}\mathrm{LE} \\
\left(\mathrm{cd} \mathrm{A}^{-1}\right)\end{array}$ & $\begin{array}{l}\mathrm{EQE} \\
(\%)\end{array}$ \\
\hline \multirow[t]{4}{*}{ Blue } & LIFT Al & 22 & 51 & 306.2 & 0.60 & 0.27 \\
\hline & LIFT Al/TBA & 21 & 48.32 & 540 & 0.89 & 0.40 \\
\hline & Convent Al & 18 & 51.16 & 25.4 & 0.05 & 0.042 \\
\hline & Convent $\mathrm{Ca}$ & 13 & 49.23 & 102.3 & 0.21 & 0.17 \\
\hline \multirow[t]{4}{*}{ Green } & LIFT Al & 26 & 37 & 134.5 & 0.36 & 0.11 \\
\hline & LIFT Al/TBA & 24 & 43 & 232.4 & 0.54 & 0.18 \\
\hline & Convent Al & 39 & 48.9 & 715.6 & 1.46 & 0.46 \\
\hline & Convent $\mathrm{Ca}$ & 22 & 44.5 & 3311 & 7.44 & 2.31 \\
\hline \multirow[t]{4}{*}{ Red } & LIFT Al & 19 & 35.67 & 84.7 & 0.24 & 0.15 \\
\hline & LIFT Al/TBA & 22 & 44.33 & 145.2 & 0.33 & 0.23 \\
\hline & Convent Al & 40 & 47.9 & 14.5 & 0.03 & 0.043 \\
\hline & Convent $\mathrm{Ca}$ & 18 & 45.14 & 726.5 & 1.64 & 2.32 \\
\hline
\end{tabular}

three colors. The interest in TBA stems from the possibility to create an adsorbed layer with a large dipole which will reduce the energy barrier to electron injection. ${ }^{22}$ In previous literature, TBA has only been applied to ITO as a holeblocking layer, ${ }^{24}$ but in this study, TBA was used to promote electron injection, giving more efficient devices. Some of the slight differences in pixel color between Figs. 2(a) and 2(b) can be accounted for by the relative differences in voltages required for a given brightness. For instance, from Table I, the LIFT Al green pixel gives only fairly low brightness $\left(134 \mathrm{~cd} \mathrm{~m}^{-2}\right)$ at $26 \mathrm{~V}$, whereas the LIFT Al/TBA green pixel gives a higher brightness $\left(230 \mathrm{~cd} \mathrm{~m}^{-2}\right)$ at a $24 \mathrm{~V}$. This can explain why the green and blue pixels in Fig. 2(a) look whiter than those in Fig. 2(b).

The addition of the iridium dyes enables phosphorescent emission, enhancing the EQE considerably by enabling EL from triplet states. ${ }^{17,18}$ The device characteristics of the pixels and conventional devices are shown in Table I and Fig. 3. When looking at the conventionally fabricated devices, the devices with calcium electrodes, Convent $\mathrm{Ca}$, show markedly increased EQE for the remarkably similar green and red $(2.31 \%$ and $2.32 \%)$ pixels relative to the blue $(0.17 \%)$. For the Convent $\mathrm{Al}$ devices, only the green $(0.46 \%)$ was increased, whilst the red $(0.043 \%)$ shows an almost identical

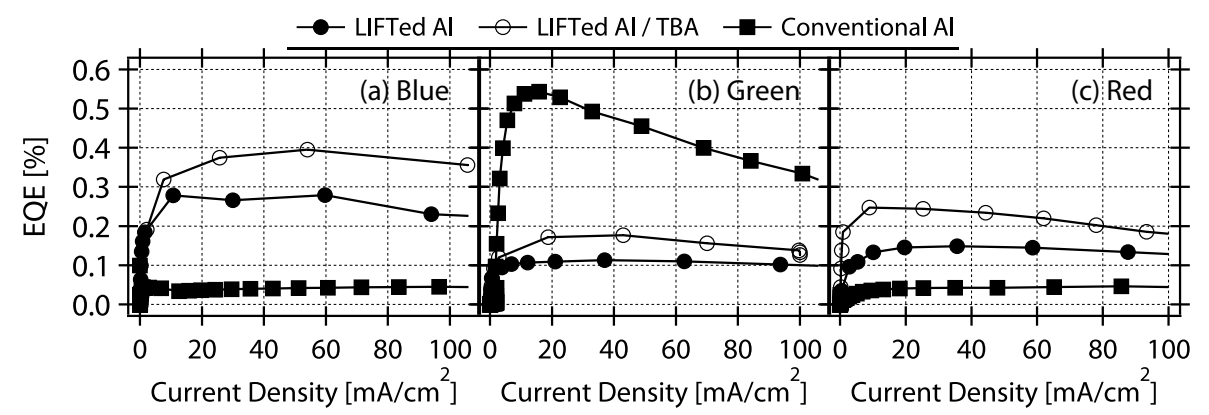

FIG. 3. The EQE plotted against current density (CD) for the LIFTed pixels (both plain $\mathrm{Al}$ and $\mathrm{Al} / \mathrm{TBA}$ ) and $\mathrm{Al}$ conventionally fabricated pixels. 
EQE to the blue $(0.042 \%)$. Additionally, both cathode types of conventionally fabricated devices show significant increases in the operating voltage when a dopant is added, but this is probably due to charge trapping by the dopants. Looking at the LIFTed pixels, both LIFT Al and LIFT All $T B A$, the undoped blue pixels are marginally more efficient than the doped green and red pixels. These EQEs are shown in Fig. 3.

A variation of the LIFT process has been used to print basic tri-color pixels based on polyfluorene. In addition, the inverse donor substrate architecture allows for direct cathode modification, and TBA has been used to demonstrate this by improving electron injection from aluminium. LIFTed pixels have been made with greater efficiencies than conventionally fabricated devices. The LIFT process uses a ns-pulsed laser to ablate a sacrificial DRL of triazene polymer with a multilayer $\mathrm{Al}$ (/TBA)/LEP stack on top. The speed of the ablation process means that LIFT has the potential to be an extremely rapid pixel deposition technique. Multilayer stacks have been transferred across a gap of $15 \mu \mathrm{m}$ at a pressure of 1 mbar to a receiver substrate consisting of glass coated with ITO/PEDOT:PSS/PVK. The multilayer stacks are transferred intact, with no fragmentation of the layers, allowing for the fabrication of functional organic semiconductor lightemitting devices. The deposited pixels have a width of $500 \mu \mathrm{m}$ and layer thicknesses of less than $100 \mathrm{~nm}$. These results demonstrate "dry" deposition of R-G-B OLED pixels across a gap to create devices of comparable functionality to those fabricated using conventional (non-patterned) OLED deposition techniques.

The authors would like to thank Ylenia Maniglio (Empa) for synthesizing the triazene polymer. Financial support is gratefully acknowledged from the FP7 European project e-LIFT (Project No. 247868 -call FP7-ICT-2009-4) and the Swiss National Science Foundation.
${ }^{1}$ C. Arnold, P. Serra, and A. Piqué, MRS Bull. 32, 23 (2007).

${ }^{2}$ N. Kattamis, N. McDaniel, S. Bernhard, and C. Arnold, Org. Electron. 12, 1152 (2011).

${ }^{3}$ S. Ko, H. Pan, S. Ryu, N. Misra, C. Grigoropoulos, and H. Park, Appl. Phys. Lett. 93, 151110 (2008).

${ }^{4}$ R. Fardel, M. Nagel, F. Nüesch, T. Lippert, and A. Wokaun, Appl. Phys. Lett. 91, 061103 (2007).

${ }^{5}$ J. Shaw-Stewart, T. Lippert, M. Nagel, F. Nüesch, and A. Wokaun, ACS Appl. Mater. Interfaces 3, 309 (2011).

${ }^{6}$ R. Friend, R. Gymer, A. Holmes, J. Burroughes, R. Marks, C. Taliani, D. Bradley, D. Dos Santos, J. Bredas, and M. Lögdlun, Nature 397, 121 (1999).

${ }^{7}$ S. Reineke, F. Lindner, G. Schwartz, N. Seidler, K. Walzer, B. Lussem, and K. Leo, Nature 459, 234 (2009).

${ }^{8}$ T. Urabe, T. Sasaoka, K. Tatsuki, and J. Takaki, SID Symp. Digest Tech. Papers 38, 161 (2007).

${ }^{9}$ M. Gather, A. Köhnen, A. Falcou, H. Becker, and K. Meerholz, Adv. Funct. Mater. 17, 191 (2007).

${ }^{10}$ S.-C. Chang, J. Liu, J. Bharathan, Y. Yang, J. Onohara, and J. Kido, Adv. Mater. 11, 734 (1999).

${ }^{11}$ B. de Gans, P. Duineveld, and U. Schubert, Adv. Mater. 16, 203 (2004).

${ }^{12}$ M. Wolk, S. Lamansky, and W. Tolbert, SID Symp. Digest Tech. Papers 39, 511 (2008).

${ }^{13}$ M. Boroson, L. Tutt, K. Nguyen, D. Preuss, M. Culver, and G. Phelan, SID Symp. Digest Tech. Papers 36, 972 (2005).

${ }^{14}$ S. Johnson, C. Bowie, B. Ivanov, H. Park, and R. Haglund, Jr., Proc. SPIE 6486, 64860G (2007).

${ }^{15}$ W. Tolbert, I.-Y. Sandy Lee, M. M. Doxtader, E. Ellis, and D. Dlott, J. Imaging Sci. Technol. 37, 411 (1993).

${ }^{16}$ S. Beaupré, P.-L. Boudreault, and M. Leclerc, Adv. Mater. 22, E6 (2010).

${ }^{17}$ Z. Chen, C. Jiang, Q. Niu, J. Peng, and Y. Cao, Org. Electron. 9, 1002 (2008).

${ }^{18}$ J.-H. Li, S. Andresen, H.-H. Liao, and Y. Yang, ACS Appl. Mater. Interfaces $\mathbf{1}, 76$ (2009).

${ }^{19}$ J. Shaw Stewart, B. Chu, T. Lippert, Y. Maniglio, M. Nagel, F. Nüesch, and A. Wokaun, Appl. Phys. A: Mater. Sci. Process. 105, 713 (2011).

${ }^{20}$ M. Nagel, R. Hany, T. Lippert, M. Molberg, F. Nüesch, and D. Rentsch, Macromol. Chem. Phys. 208, 277 (2007).

${ }^{21}$ J. Wang, R. Auyeung, H. Kim, N. Charipar, and A. Piqué, Adv. Mater. 22, 4462 (2010).

${ }^{22}$ F. Nüesch, L. Rothberg, E. Forsythe, Q. Toan Le, and Y. Gao, Appl. Phys. Lett. 74, 880 (1999).

${ }^{23}$ T. Ferenczi, M. Sims, and D. Bradley, J. Phys.: Condens. Matter 20, 045220 (2008).

${ }^{24}$ F. Nüesch, Y. Li, and L. Rothberg, Appl. Phys. Lett. 75, 1799 (1999). 